\title{
A importância da narração de histórias na educação em enfermagem
}

\author{
The importance of storytelling in nursing education \\ La importancia de contar historias en la educación en enfermeira
}

Recebido: 11/11/2021 | Revisado: 19/11/2021 | Aceito: 25/11/2021 | Publicado: 03/12/2021

Jéssica Mayara da Silva Araújo

ORCID: https://orcid.org/0000-0003-0453-4416

Universidade Tiradentes, Brasil

E-mail: jessicamayara3357@gmail.com

Maria Mylena Gomes Santos

ORCID: https://orcid.org/0000-0002-9555-8175

Universidade Tiradentes, Brasil

E-mail: myly.myh@hotmail.com

Queila Samara dos Santos Farias

ORCID: https://orcid.org/0000-0002-4686-4539 Universidade Tiradentes, Brasil E-mail: queilasamara20@gmail.com

Renata Santos da Silva

ORCID: https://orcid.org/0000-0001-5459-3444 Universidade Tiradentes, Brasil

E-mail: renatasilvasts@yahoo.com

Fernanda Dantas Barros

ORCID: https://orcid.org/0000-0001-9155-1665 Universidade Tiradentes, Brasil

E-mail: nanda-dantas@hotmail.com

Manuela de Carvalho Vieira Martins ORCID: https://orcid.org/0000-0003-1222-5955 Universidade Tiradentes, Brasil E-mail: manuela.cvm@hotmail.com

Fernanda Costa Martins Gallotti

ORCID: https://orcid.org/0000-0002-9063-1273 Universidade Tiradentes, Brasil

E-mail: fernanda.gallotti@souunit.com.br

\begin{abstract}
Resumo
Este estudo tem como objetivo identificar as evidências disponíveis na literatura sobre a utilização da narração de histórias como estratégia de aprendizagem na formação do enfermeiro. Trata-se de uma revisão de escopo com metodologia do Instituto Joanna Briggs, realizada nas bases de dados PubMed, Scielo, ERIC, Science Direct e Lilacs, utilizando os operadores booleanos "AND" e "OR". Foram revisados estudos originais publicados até agosto de 2021. Das 42 produções analisadas na íntegra, 21 compuseram a amostra, e foram publicadas entre 1998 e 2020 em seis países, incluindo Reino Unido, Estados Unidos da América, Austrália, Canadá, Noruega e Suécia. Entre os tipos de pesquisa incluídos, destacaram-se estudos qualitativos $(14,3 \%)$ e descritivos $(14,3 \%)$. Quanto a temática das narrações identificou-se experiências clínicas $(42,9 \%)$ e histórias dos pacientes (19\%) como as mais abordadas, e o recurso mais utilizado foram as mídias digitais $(53,6 \%)$. Evidenciou-se benefícios do uso da narração de histórias na educação em enfermagem, entre eles: desenvolvimento e/ou aumento da empatia, captação da atenção, formação do elo teoria-prática, melhora da compreensão e capacitação. Essas contribuições possibilitam uma formação diferencial do profissional, proporcionando uma visão integral, diferenciada e humanizada do paciente. Entretanto, algumas desvantagens estão associadas a seu uso, como vulnerabilidade do contador de histórias e desconforto devido ao impacto emocional. As evidências demonstram a aplicabilidade da metodologia, sendo atributos importantes para a sua efetividade o conteúdo da narrativa e os recursos utilizados. Sugere-se que pesquisas futuras ampliem suas amostras estudadas, a fim de compor uma base sólida que promova a validação dessa ferramenta.
\end{abstract}

Palavras-chave: Aprendizagem; Educação em enfermagem; Ensino; Narração.

\begin{abstract}
This study aims to identify the evidence available in the literature on the use of storytelling as a learning strategy in nursing education. This is a scope review using the Joanna Briggs Institute methodology, carried out in the PubMed, Scielo, ERIC, Science Direct and Lilacs databases, using the Boolean operators "AND" and "OR". Original studies published until August 2021 were reviewed. Of the 42 productions analyzed in full, 21 comprised the sample, and were published between 1998 and 2020 in six countries, including the United Kingdom, United States of America, Australia, Canada, Norway and Sweden. Among the types of research included, qualitative (14.3\%) and descriptive (14.3\%)
\end{abstract}


studies stood out. As for the theme of the narratives, clinical experiences (42.9\%) and patients' stories (19\%) were identified as the most discussed, and the most used resource was digital media (53.6\%). Benefits of the use of storytelling in nursing education were evidenced, including: developing and/or increasing empathy, attracting attention, forming the theory-practice link, improving understanding and training. These contributions enable a differential training of the professional, providing a comprehensive, differentiated and humanized view of the patient. However, some disadvantages are associated with its use, such as the storyteller's vulnerability and discomfort due to the emotional impact. The evidence demonstrates the applicability of the methodology, with the narrative content and the resources used being important attributes for its effectiveness. It is suggested that future researches expand their studied samples in order to compose a solid base that promotes the validation of this tool.

Keywords: Learning; Nursing education; Teaching; Narration.

\section{Resumen}

Este estudio tiene como objetivo identificar la evidencia disponible en la literatura sobre el uso del storytelling como estrategia de aprendizaje en la educación en enfermería. Se trata de una revisión del alcance utilizando la metodología del Joanna Briggs Institute, realizada en las bases de datos PubMed, Scielo, ERIC, Science Direct y Lilacs, utilizando los operadores booleanos "AND" y "OR". Se revisaron los estudios originales publicados hasta agosto de 2021. De las 42 producciones analizadas en su totalidad, 21 conformaron la muestra, y fueron publicadas entre 1998 y 2020 en seis países, entre ellos Reino Unido, Estados Unidos de América, Australia, Canadá, Noruega y Suecia. Entre los tipos de investigación incluidos, se destacaron los estudios cualitativos $(14,3 \%)$ y descriptivos $(14,3 \%)$. En cuanto al tema de las narrativas, las experiencias clínicas $(42,9 \%)$ y las historias de los pacientes (19\%) fueron identificadas como las más discutidas, y el recurso más utilizado fueron los medios digitales $(53,6 \%)$. Se evidenciaron beneficios del uso del storytelling en la educación de enfermería, entre ellos: desarrollar y / o incrementar la empatía, llamar la atención, formar el vínculo teoría-práctica, mejorar la comprensión y la formación. Estas aportaciones posibilitan una formación diferencial del profesional, aportando una visión integral, diferenciada y humanizada del paciente. Sin embargo, algunas desventajas están asociadas a su uso, como la vulnerabilidad y el malestar del narrador debido al impacto emocional. La evidencia demuestra la aplicabilidad de la metodología, siendo el contenido narrativo y los recursos utilizados atributos importantes para su efectividad. Se sugiere que futuras investigaciones amplíen sus muestras estudiadas con el fin de componer una base sólida que promueva la validación de esta herramienta.

Palabras clave: Aprendizaje; Educación en enfermería; Enseñanza; Narración.

\section{Introdução}

Como uma das primeiras formas de comunicação do ser humano, o ato de contar histórias tem sido empregado no intuito de transmitir informação e conhecimento entre gerações (Santos, 2020). No contexto educacional, a narração de histórias também conhecida por Storytelling atua como uma poderosa ferramenta cujo objetivo é compartilhar conhecimento para propiciar aprendizagem ao aluno. Seu uso amplia o desenvolvimento de sentimento empático com o enredo da narrativa, estimulando a busca de resolução para a problemática, evento ou situação apresentada (Camargo \& Daros, 2018).

Para Collins (2021), a mesma permite relacionar o conteúdo da sala de aula com situações reais e históricas, vivenciadas por pesquisadores e intelectuais que contribuíram significativamente para a sociedade. Desse modo, pode operar como fonte de inspiração, instigando o aluno a buscar grandes realizações profissionais, bem como assimilar os erros cometidos em experiências passadas a fim de torná-lo ávido pela aprendizagem, porém cauteloso em suas ações.

A narração de histórias é considerada uma das metodologias ativas de ensino-aprendizagem, visto que pressupõe uma reação dos ouvintes à narrativa, de modo que os mesmos participem conjunta e ativamente da construção da aprendizagem (Valença \& Tostes, 2019). No ensino em saúde, nota-se a necessidade de incorporação dessas metodologias para que sejam estabelecidas diretrizes inovadoras para conectar teoria e prática e romper a dicotomia existente entre a formação clínica e o ensino básico (Roman et al., 2017).

Essa necessidade não é recente, e tem sido abordada desde 2001 através das Diretrizes Curriculares Nacionais (DCNs) dos cursos da saúde, que demonstram o requerimento de formação crítica e reflexiva dos profissionais, com o emprego de metodologias estimuladoras da reflexão acerca da realidade social. O objetivo é dotar o profissional de conhecimentos para exercer competências e habilidades gerais, tais como atenção à saúde, tomada de decisões, comunicação, liderança, 
administração, gerenciamento e educação permanente, de forma a colocá-los em ação para resolver problemas e enfrentar situações inesperadas (Brasil, 2001).

A convergência dos objetivos das DCNs com os da narração de histórias para a resolução de problemas proporciona potencial destaque para a utilização dessa metodologia no ensino em enfermagem. Isso é reforçado pela Resolução nº 573 , de 31 de janeiro de 2018, que traz em seu Art. $5^{\circ}$ as metodologias ativas de ensino-aprendizagem como um dos fundamentos para propiciar ao enfermeiro um perfil profissional humanista, para atuar com qualidade, efetividade e resolubilidade no SUS (Brasil, 2018).

Como vantagens da mesma, têm-se a variedade de elementos que são utilizados para empregar a estratégia, o que inclui a oralidade, música, teatro e tecnologias com recursos audiovisuais (Santos, 2020). Com o avanço das tecnologias, vem sendo bastante utilizada a narrativa digital, definida como uma apresentação multimídia de uma história, geralmente em formato de vídeo (Lambert \& Hessler, 2018). Além desse formato, pode-se encontrar filmes curtos com imagens, slideshows para apoio de performance e narração de histórias e videoclipes (Mclellan, 2007).

A perspectiva de maior uso das tecnologias no ensino advindas da adoção de modelos de educação remota, em decorrência da pandemia pela Coronavírus Disease 2019 (COVID-19) (Saraiva, Traversini, \& Lockmann, 2020), torna a narrativa digital promissora. Dessa forma, garante-se a aplicabilidade da narração de histórias, com o intuito de proporcionar uma visão da prática clínica, sendo de especial importância quando há limitação da práxis ao aluno (Treloar, Mcmillan, \& Stone, 2017).

Este estudo justifica-se pelo potencial contribuição do uso da narração de histórias na educação e no desenvolvimento de determinadas habilidades e competências necessárias no processo de formação do enfermeiro. Desse modo, se faz necessário buscar na literatura evidências quanto ao uso dessa metodologia ativa na formação em enfermagem, uma vez que favorece tanto o discente quanto o narrador docente no processo de reflexão e autoconsciência (Edwards, 2016).

Nesta conjuntura, formulou-se a seguinte questão norteadora: Qual (is) a (s) evidência (s) acerca da utilização da narração de histórias como estratégia de aprendizagem na formação do enfermeiro? Com o intuito de responder aos questionamentos propostos, esta pesquisa tem por objetivo identificar as evidências disponíveis na literatura sobre a utilização da narração de histórias como estratégia de aprendizagem na formação do enfermeiro.

\section{Metodologia}

Trata-se de uma revisão de escopo, desenvolvida de acordo com a metodologia do Instituto Joanna Briggs. Optou-se pela realização de um estudo do tipo scoping review, por seu uso ser indicado quando um corpo de literatura não passou por uma revisão de forma abrangente ou apresenta uma estrutura grande, complexa ou heterogênea não sendo apta a uma revisão sistemática mais precisa (Peters et al., 2015). Essa pesquisa foi norteada pelo protocolo Preferred Reporting Items for Systematic Reviews and Meta-Analyses extension for scoping review (PRISMA-ScR) (Tricco et al., 2018).

\section{Critérios de elegibilidade e estratégia de busca}

Para estruturação das estratégias de busca utilizou-se o Método PCC (P: População, C: Conceito, C: Contexto), para melhor formulação da pergunta norteadora desta pesquisa. Dessa forma, a pergunta de investigação da Scoping Review foi: Qual (is) a (s) evidência (s) acerca da utilização da narração de histórias como estratégia de aprendizagem na formação do enfermeiro?

A busca foi realizada nas bases de dados: PubMed, Latin American and Caribbean Center on Health Sciences Information (Lilacs), Scientific Electronic Library Online (Scielo), Education Resources Information Center (ERIC) e Science Direct. A estratégia de busca foi ajustada de acordo com os protocolos de cada base e os descritores para combinações foram 
definidos a partir do vocabulário controlado da Medical Subject Headings (MeSH), com o uso dos operadores Booleanos “AND” e “OR”, sendo utilizados: Educação em enfermagem; Educational Technology; Narration; Learning; Aprendizagem.

De modo a explorar a estratégia de busca, termos não padronizados foram adicionados. A estratégia completa por base de dados incluída na pesquisa foi descrita no Quadro 1.

Quadro 1. Expressões de busca utilizadas nas bases de dados incluídas no estudo, 2021.

\begin{tabular}{|l|l|}
\hline \multicolumn{1}{|c|}{ Base de dados } & \multicolumn{1}{c|}{ Estratégia de busca } \\
\hline PubMed & $\begin{array}{l}((((\text { Narrative medicine) OR (Narration)) OR (storytelling)) AND (Educational Technology)) AND } \\
\text { (Learning)) AND (Nursing) }\end{array}$ \\
\hline Lilacs & $\begin{array}{l}\text { (Narration) OR (História em Quadrinhos) AND (Educação em enfermagem) AND (Aprendizagem) OR } \\
\text { (Learning) }\end{array}$ \\
\hline ERIC & $\begin{array}{l}\text { (Narration) OR (Storytelling) OR (narrative medicine) AND (Education) OR (Nursing) AND } \\
\text { (Learning) }\end{array}$ \\
\hline SCIELO & $\begin{array}{l}\text { (Narration) OR (Narrativa) OR (História em Quadrinhos) AND (Educação em enfermagem) AND } \\
\text { (Aprendizagem) OR (Learning) }\end{array}$ \\
\hline Science Direct & $\begin{array}{l}((((\text { Narrative medicine) OR (Narration)) OR (storytelling)) AND (Educational Technology)) AND } \\
\text { (Learning) AND (Nursing) }\end{array}$ \\
\hline
\end{tabular}

Fonte: Autores (2021).

Os critérios de inclusão aplicados nesta pesquisa foram: estudos originais, publicados de 1998 até agosto de 2021, em português, espanhol ou inglês, que apresentassem como objeto de investigação a aplicação de narrativas como estratégia de ensino aprendizagem nos cursos de enfermagem. Foram excluídos os estudos: não disponíveis na íntegra, cuja solicitação de arquivo não foi respondida pelo autor e publicações em congressos. A busca dos estudos foi realizada em setembro de 2021.

\section{Seleção dos artigos e coleta dos dados}

Os títulos dos estudos foram organizados em planilha do software Microsoft Excel by Office 2019 e com uso do gerenciador de referências Mendeley, foi realizada a identificação e exclusão dos arquivos em duplicatas, seguida da exclusão de trabalhos duplicados identificados manualmente.

A seleção mediante critérios de elegibilidade foi realizada por dois revisores (J.M.S.A. e M.M.G.S), de forma independente. Os estudos que geraram alguma discordância entre os revisores quanto à inclusão foram discutidos com um terceiro revisor (F.C.M.G).

Após triagem por título, resumo e leitura na íntegra, os artigos selecionados foram analisados pelos revisores e extraídas as seguintes informações: autores, ano de publicação, país de realização do estudo, título, tipo de estudo, número de estudantes inseridos no estudo, período do curso de graduação ou especialização, objetivo, tema e características da narração e os principais resultados envolvidos nas pesquisas.

Em casos de divergência na leitura do texto completo entre os revisores, reuniões eram realizadas em busca de um consenso. Caso a pesquisa fosse considerada inelegível, a razão primária da exclusão foi registrada para composição do fluxo de seleção dos artigos.

\section{Análise dos dados}

Os dados extraídos dos estudos incluídos na Scoping Review foram apresentados por meio de análise descritiva das principais características dos estudos, visando apontar a relação entre a contação de histórias e a retenção da aprendizagem. 


\section{Resultados}

A partir de uma triagem inicial, foram identificados 1.765 estudos, sendo 30,8\% indexados de forma simultânea em duas ou mais bases de dados. A triagem foi sequenciada de forma criteriosa com a leitura dos títulos e resumos resultando em 42 artigos para leitura na íntegra. O processo de identificação dos estudos encontra-se ilustrado na Figura 1.

Figura 1. Processo de identificação dos estudos - PRISMA-ScR Diagram flow, 2021.

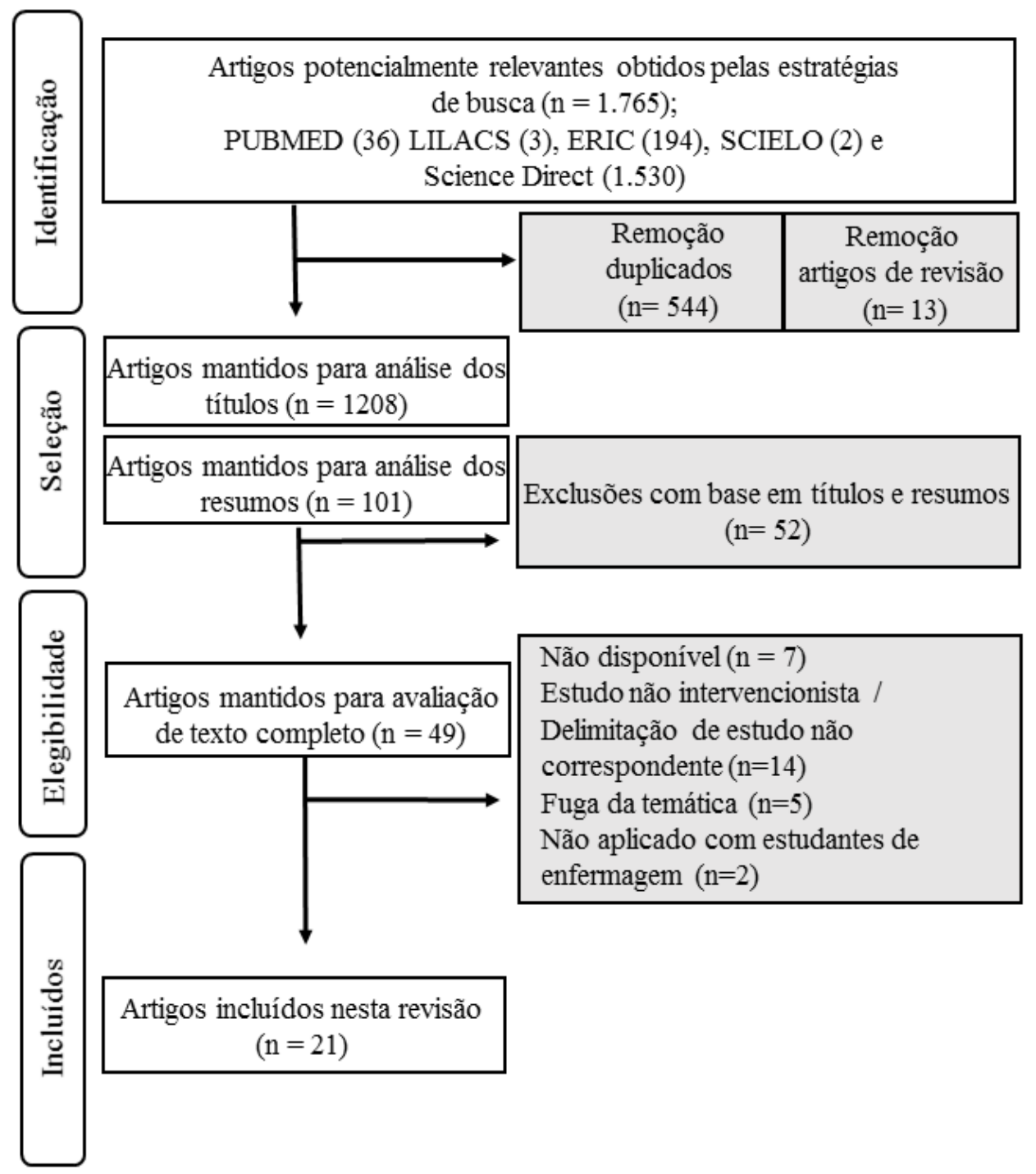

Fonte: Dados da pesquisa (2021).

No que diz respeito à leitura na íntegra dos estudos, observou-se que 28 publicações não atendiam ao objetivo requerido para a presente pesquisa. Os motivos que levaram à exclusão dos mesmos incluíram a indisponibilidade na íntegra, o design do estudo, a fuga da temática e a ausência de relação com a enfermagem. O detalhamento da exclusão de cada estudo encontra-se disposto na Tabela 1. 
Tabela 1. Caracterização dos estudos excluídos após leitura na íntegra, 2021.

\begin{tabular}{|c|c|c|}
\hline Autores/Ano & Título & Motivo da exclusão \\
\hline SHERWOOD, 2021. & $\begin{array}{l}\text { Quality and Safety Education for Nurses: Making progress in patient safety, learning } \\
\text { from COVID-19. }\end{array}$ & $\begin{array}{l}\text { Não aborda a narração de } \\
\text { histórias. }\end{array}$ \\
\hline $\begin{array}{l}\text { PETTY; JARVIS; THOMAS, } \\
2020 .\end{array}$ & $\begin{array}{c}\text { Exploring the impact of digital stories on empathic learning in neonatal nurse } \\
\text { education. }\end{array}$ & Indisponível na íntegra. \\
\hline O'CONNOR et al., 2020. & Podcasting in nursing and midwifery education: An integrative review. & Design do estudo. \\
\hline MOORE; MILLER, 2020. & Video Storytelling in the Classroom: The Role of Narrative Transportation. & Indisponível na íntegra. \\
\hline $\begin{array}{l}\text { YOUNG; GODBOLD; } \\
\text { WOOD, } 2019 .\end{array}$ & Nurses' experiences of learning to care in practice environments: A qualitative study. & $\begin{array}{l}\text { Não aborda a narração de } \\
\text { histórias. }\end{array}$ \\
\hline WU et al., 2019. & Narrative Approach to Goals of Care Discussions: A Novel Curriculum. & $\begin{array}{l}\text { Não se relaciona à } \\
\text { enfermagem. }\end{array}$ \\
\hline WESTBURY, 2018. & $\begin{array}{l}\text { Use of Guided Reflection Tools in Collaborative Health Care Settings Following } \\
\text { Simulated Learning. }\end{array}$ & Não se encaixa na revisão. \\
\hline TOMASINO, 2018. & $\begin{array}{c}\text { Nurse Educators Utilizing Narratives with Technology to Improve the Student } \\
\text { Learning Experience. }\end{array}$ & Indisponível na íntegra. \\
\hline $\begin{array}{l}\text { HAMPTON; PEARCE; } \\
\text { MOSER, } 2017 .\end{array}$ & Preferred methods of learning for nursing students in an on-line degree program. & $\begin{array}{l}\text { Não aborda a narração de } \\
\text { histórias. }\end{array}$ \\
\hline $\begin{array}{l}\text { FOWLER; BAKER; } \\
\text { GERAGHTY, } 2017 .\end{array}$ & $\begin{array}{l}\text { Is faculty practice valuable? The experience of Western Australian nursing and } \\
\text { midwifery academics undertaking faculty clinical practice - A discussion paper. }\end{array}$ & Design do estudo. \\
\hline WOLF et al., 2017. & $\begin{array}{l}\text { The design process of a multimodal module that synthesized knowledge } \\
\text { across nursing courses. }\end{array}$ & $\begin{array}{l}\text { Não aborda a narração de } \\
\text { histórias. }\end{array}$ \\
\hline PALIADELIS; WOOD, 2016. & $\begin{array}{l}\text { Learning from clinical placement experience: Analysing nursing students' final } \\
\text { reflections in a digital storytelling activity. }\end{array}$ & Não se encaixa na revisão. \\
\hline WILSON; WALKER, 2016. & $\begin{array}{l}\text { Turning a crisis into an interactive drama: An introductory paper of a 'clickers theatre' } \\
\text { in nurse education. }\end{array}$ & Não se encaixa na revisão. \\
\hline $\begin{array}{l}\text { LEVETT-JONES; BOWEN; } \\
\text { MORRIS, } 2015 .\end{array}$ & $\begin{array}{l}\text { Enhancing nursing students' understanding of threshold concepts through the use of } \\
\text { digital stories and a virtual community called 'Wiimali'. }\end{array}$ & Design do estudo. \\
\hline GILLHAM et al., 2015. & CaseWorld ${ }^{\mathrm{TM}}$ : Interactive, media rich, multidisciplinary case based learning. & $\begin{array}{l}\text { Não aborda a narração de } \\
\text { histórias. }\end{array}$ \\
\hline MARUXO et al., 2015. & $\begin{array}{c}\text { Webquest e história em quadrinhos na formação de recursos humanos em } \\
\text { Enfermagem. }\end{array}$ & Não se encaixa na revisão. \\
\hline WEBB-CORBETT et al., 2013. & $\begin{array}{c}\text { New media simulation stories in nursing education: a quasi-experimental study } \\
\text { exploring learning outcomes. }\end{array}$ & Indisponível na íntegra. \\
\hline COLOMER et al., 2013. & Reflective learning in higher education: A comparative analysis. & Não se encaixa na revisão. \\
\hline OSMOND et al., 2012. & An Open Letter to Our Future Students in "Narrative and the Caring Professions". & Design do estudo. \\
\hline HAIGH; HARDY, 2011. & Tell me a story - a conceptual exploration of storytelling in healthcare education. & Design do estudo. \\
\hline SNELSON, 2010 & Stories of exemplary hospital registered nurses: A narrative analysis. & Não se encaixa na revisão. \\
\hline $\begin{array}{l}\text { STRANIERI; YEARWOOD, } \\
2008 .\end{array}$ & $\begin{array}{c}\text { Enhancing Learning Outcomes with an Interactive Knowledge-Based Learning } \\
\text { Environment Providing Narrative Feedback. }\end{array}$ & Design do estudo. \\
\hline SIERPINA et al., 2007. & Regaining Our Humanity Through Story. & $\begin{array}{l}\text { Não se relaciona à } \\
\text { enfermagem. }\end{array}$ \\
\hline $\begin{array}{l}\text { YEARWOOD; STRANIERI, } \\
2007 .\end{array}$ & Narrative-Based Interactive Learning Environments from Modelling Reasoning. & Indisponível na íntegra. \\
\hline IRONSIDE, 2003. & $\begin{array}{l}\text { New pedagogies for teaching thinking: the lived experiences of students and teachers } \\
\text { enacting narrative pedagogy. }\end{array}$ & Indisponível na íntegra. \\
\hline ANDREWS et al., 2001. & Enacting Narrative Pedagogy. The Lived Experiences of Students and Teachers. & Indisponível na íntegra. \\
\hline BOWLES, 1995. & Storytelling: a search for meaning within nursing practice. & Design do estudo. \\
\hline WEISBERG; DUFFIN, 1995. & $\begin{array}{l}\text { Evoking the Moral Imagination: Using Stories to Teach Ethics and Professionalism to } \\
\text { Nursing, Medical, and Law Students. }\end{array}$ & Design do estudo. \\
\hline
\end{tabular}

Fonte: Autores (2021).

As características quanto ao ano e local de realização da pesquisa foram apresentadas na Figura 2. Observa-se que as publicações foram realizadas no período de 1998 a 2020 e publicados em seis países diferentes, incluindo Reino Unido (38\%), Estados Unidos da América (28,6\%), Austrália (14,3\%), Canadá (9,5\%), Noruega (4,8\%) e Suécia (4,8\%). 
Figura 2. Publicações por ano e país. (A) Número de artigos por ano; (B) Número de artigos por país.

A

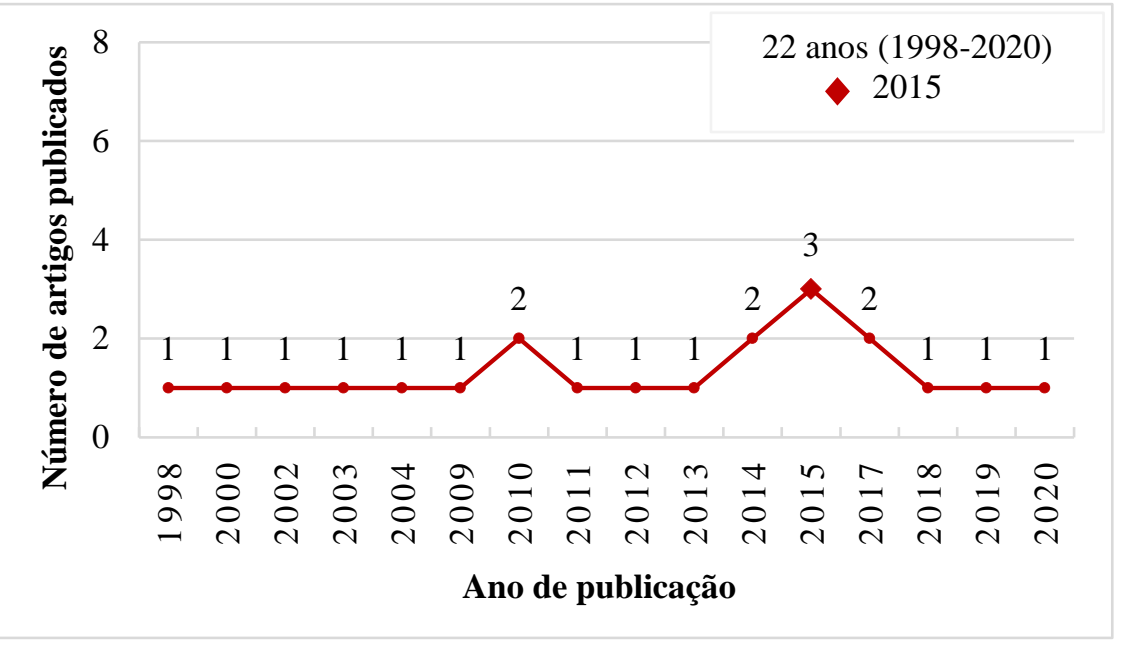

B

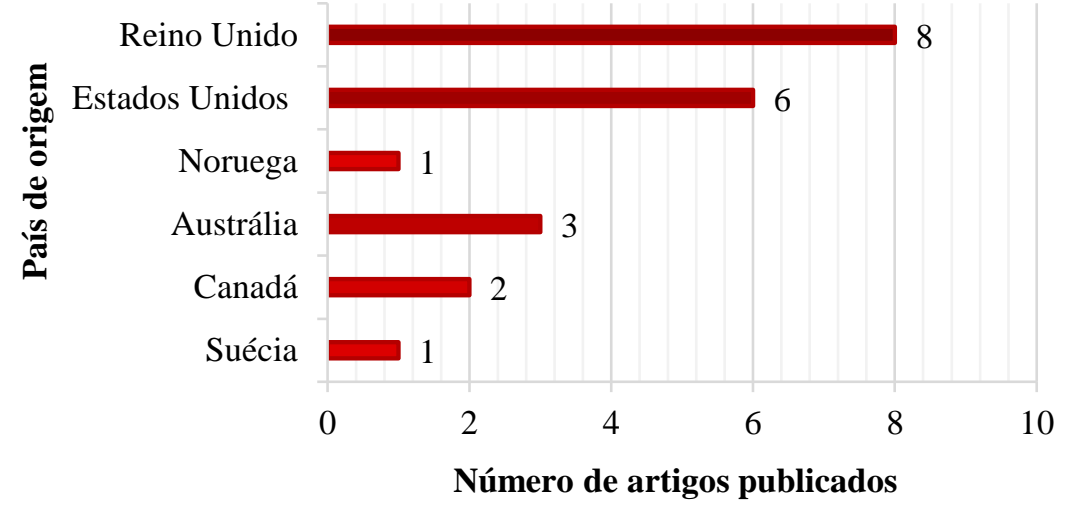

Fonte: Autores (2021).

Em relação ao tipo de pesquisa realizada, destacaram-se os estudos qualitativos (14,3\%) e descritivos (14,3\%). A análise temática das narrações de histórias possibilitou identificar as experiências clínicas (42,9\%) e as histórias dos pacientes (19\%) como os temas mais abordados no uso da metodologia. No que diz respeito aos recursos utilizados para empregar a estratégia, notou-se uma grande variabilidade, tendo alguns estudos aplicado mais de um em seus métodos de pesquisa. O tipo de meio mais prevalente foram as mídias digitais $(53,6 \%)$. Esses dados encontram-se dispostos na Tabela 2, juntamente com as especificações das mídias. 
Tabela 2. Distribuição percentual de tipos de pesquisa, temas abordados e recursos utilizados, 2021.

\begin{tabular}{|c|c|}
\hline VARIÁVEIS & $\mathbf{N}(\%)$ \\
\hline \multicolumn{2}{|l|}{ Tipo de pesquisa } \\
\hline Qualitativa & $3(14,3)$ \\
\hline Descritiva & $3(14,3)$ \\
\hline Estudo fenomenológico & $2(9,5)$ \\
\hline Pesquisa-ação & $2(9,5)$ \\
\hline Estudo piloto & $2(9,5)$ \\
\hline Estudo quase-experimental & $1(4,8)$ \\
\hline Estudo fenomenográfico & $1(4,8)$ \\
\hline Estudo de coorte & $1(4,8)$ \\
\hline Estudo duplo & $1(4,8)$ \\
\hline Relato de desenvolvimento & $1(4,8)$ \\
\hline Pesquisa experimental & $1(4,8)$ \\
\hline ND & $3(14,3)$ \\
\hline \multicolumn{2}{|l|}{ Tema da narração } \\
\hline Cardiologia & $1(4,8)$ \\
\hline Questões contemporâneas em saúde & $1(4,8)$ \\
\hline Cuidados paliativos e/ou no fim da vida & $2(9,5)$ \\
\hline Histórias do paciente & $4(19)$ \\
\hline Obstetrícia & $1(4,8)$ \\
\hline Experiências clínicas & $9(42,9)$ \\
\hline Saúde mental & $1(4,8)$ \\
\hline Conceito de fisiopatologia & $1(4,8)$ \\
\hline ND & $1(4,8)$ \\
\hline \multicolumn{2}{|l|}{ Recurso utilizado } \\
\hline Filme & $2(8)$ \\
\hline Podcast & $1(3,8)$ \\
\hline Simulação filmada & $1(3,8)$ \\
\hline Livro de história com fotos & $1(3,8)$ \\
\hline Gravação audiovisual & $1(3,8)$ \\
\hline Interativas e baseadas na $W e b$ & $1(3,8)$ \\
\hline Aplicativo & $1(3,8)$ \\
\hline Objeto virtual de aprendizagem & $1(3,8)$ \\
\hline Curta-metragem & $1(3,8)$ \\
\hline Apresentação multimídia c/ imagens e músicas & $1(3,8)$ \\
\hline Dramas de áudio & $1(3,8)$ \\
\hline Mídia digital não especificada & $1(3,8)$ \\
\hline Livros & $1(3,8)$ \\
\hline Escrita & $4(15,4)$ \\
\hline Dramatização & $1(3,8)$ \\
\hline Verbal & $6(23,1)$ \\
\hline
\end{tabular}

ND - Não descrito. Fonte: Autores (2021).

Os dados extraídos dos estudos, relacionados aos elementos que compuseram as amostras utilizadas nas pesquisas, incluem, o número de participantes, programa de formação e semestre do curso. Os mesmos encontram-se dispostos na Tabela 3, juntamente com a autoria, características e principais resultados encontrados na aplicação das narrações de histórias em estudantes de enfermagem, sendo estes alguns dos focos de investigação deste estudo. 
Relacionado ao quantitativo total de participantes das pesquisas, como demonstrado na Tabela 3, houve uma variação de 7 a 385 participantes, com um total geral de 1.339 que incluem estudantes de enfermagem e de outras áreas como educação, e enfermeiros. Dos estudos analisados, nove envolvem alunos graduandos no curso de enfermagem, cinco de especialização/pósgraduação para enfermeiros, um de curso de preceptoria para enfermeiros e seis não foram descritos.

Através da análise dos artigos, ainda é possível verificar na Tabela 3 que houve concordância dos autores na maioria dos resultados encontrados na aplicação das narrações de histórias. Essas concordâncias relacionam-se principalmente com o desenvolvimento de sentimento empático. 


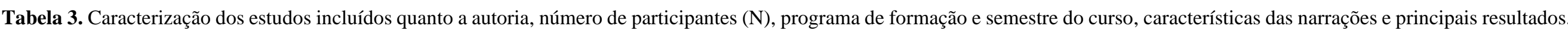

\begin{tabular}{|c|c|c|c|c|}
\hline Referência & $\mathbf{N}$ & $\begin{array}{l}\text { Programa de formação; } \\
\text { Período do curso }\end{array}$ & Características da narração & Principais resultados \\
\hline $\begin{array}{l}\text { Conlon, Smart, \& } \\
\text { Mcintosh, } 2020 .\end{array}$ & 7 & $\begin{array}{l}\text { Especialização Saúde } \\
\text { Mental; ND. }\end{array}$ & $\begin{array}{l}\text { Foram usadas: simulação filmada com } \\
\text { atores amadores ( } 15 \text { min.); narrativa fictícia } \\
\text { usando livro de histórias com fotos ( } 20 \\
\text { min.); filme de narrativa fictícia com atores } \\
\text { profissionais ( } 1 \text { h.) e podcast de áudio ( } 3 \\
\text { min.). }\end{array}$ & $\begin{array}{l}\text { - O filme foi considerado mais autêntico, seguido pelo livro de histórias e a } \\
\text { simulação; } \\
\text { - } \quad \text { O podcast teve o pior desempenho, pela falta de relevância e curta duração; } \\
\text { As interpretações dos cenários variavam de acordo com as realidades dos } \\
\text { - } \quad \text { As histicírias; }\end{array}$ \\
\hline Beck \& Sitzman, 2019. & 12 & ND. & $\begin{array}{l}\text { As histórias eram digitais, através de } \\
\text { mídias. }\end{array}$ & $\begin{array}{l}\text { - O uso da mídia capta a atenção dos estudantes devido ao grande uso de tecnologias } \\
\text { pelos mesmos; } \\
\text { - Justifica-se o uso de histórias digitais para superar a monotonia e promover } \\
\text { experiências de compartilhamento; } \\
\text { - Acredita-se que ver e ouvir histórias digitais provoca sentimentos, contribuindo para } \\
\text { o emocional afetivo. }\end{array}$ \\
\hline Urstad et al., 2018. & 37 & $\begin{array}{l}\text { Graduação; primeiro ano } \\
\text { (17) e terceiro ano (20). }\end{array}$ & $\begin{array}{l}\text { Foram histórias digitais em formato de } \\
\text { filme contendo as vozes dos alunos. }\end{array}$ & $\begin{array}{l}\text { Ocorreu forte envolvimento emocional e compreensão mais profunda, favorecendo } \\
\text { engajamento e novos insights; } \\
\text { Os efeitos multimídia pareceram criar uma dimensão extra da realidade, senso de } \\
\text { relevância e proximidade de uma situação real; } \\
\text { - Os alunos sentiram propriedade e liberdade na reflexão gerada; } \\
\text { Estar no papel de contador provocou vulnerabilidade, cultura de aprendizagem } \\
\text { respeitosa e tolerante. }\end{array}$ \\
\hline $\begin{array}{l}\text { Johnston, Parker, \& } \\
\text { Fox, } 2017 .\end{array}$ & 385 & Graduação; terceiro ano. & $\begin{array}{l}\text { Realizada através de uma gravação } \\
\text { audiovisual num pré-brief de simulação. }\end{array}$ & $\begin{array}{l}\text { - Aumento estatístico significativo no grupo intervenção acerca da confiança em ir } \\
\text { para o ambiente clínico real; } \\
\text { - Melhora da competência clínica e preparo para atuar no ambiente clínico "da vida } \\
\text { real"; } \\
\text { - Não houve interferência estatística significativa no realismo e valor da simulação, } \\
\text { apesar das pontuações médias aumentadas no grupo intervenção. }\end{array}$ \\
\hline $\begin{array}{l}\text { Scamell \& Hanley, } \\
2017 .\end{array}$ & 111 & $\begin{array}{l}\text { Especialização } \\
\text { Obstetrícia; primeiro ano. }\end{array}$ & $\begin{array}{l}\text { As narrações são interativas e baseadas na } \\
\text { web, através de duas comunidades fictícias } \\
\text { e virtuais criadas na plataforma Moodle. }\end{array}$ & $\begin{array}{l}\text { Houve aprendizagem cooperativa e envolvimento no cuidado na visão da mulher e } \\
\text { do profissional; } \\
\text { - Identificou-se a qualidade da assistência explorando a ansiedade que a prática } \\
\text { antipática causa e discutindo sobre sensibilidade e empatia; } \\
\text { - O ambiente narrativo foi considerado um espaço confortável; } \\
\text { - } \quad \text { Houve aumento da compreensão profissional da prática da obstetrícia; } \\
\text { Alguns alunos sentiram que a abordagem não melhorou a aprendizagem. }\end{array}$ \\
\hline $\begin{array}{l}\text { Price, Strodtman, } \\
\text { Brough, Lonn, \& Luo, } \\
2015 .\end{array}$ & 134 & Graduação; quarto ano. & $\begin{array}{l}\text { Foram criadas pelos alunos no aplicativo } \\
\text { VoiceThread com duração de } 5 \text { minutos, a } \\
\text { partir de uma pré-aprendizagem e histórias } \\
\text { pessoais e experiências compartilhadas por } \\
\text { pacientes/familiares. }\end{array}$ & $\begin{array}{l}\text { - } \quad \text { Demonstração de amplitude nas histórias e aplicação de conceitos; } \\
\text { - } \quad \text { Promoção de novos insights e auxilio para aplicar o conteúdo à prática; } \\
\text { aumentando a compreensão de conceitos; } \\
\text { - Houve aprendizagem sobre cultura, espiritualidade e singularidade das experiências; } \\
\text { Os alunos gostaram da criatividade e flexibilidade da narrativa, mas alguns } \\
\text { acreditam que não houve aprendizagem profunda. }\end{array}$ \\
\hline
\end{tabular}




\begin{tabular}{|c|c|c|c|c|}
\hline $\begin{array}{l}\text { Hold, Blake, \& Ward, } \\
2015 .\end{array}$ & 19 & Graduação; ND. & $\begin{array}{l}\text { Foi realizada verbalmente através de } \\
\text { palestras. }\end{array}$ & $\begin{array}{l}\text { - Ocorreu ganho de conhecimento e percepção através das histórias dos palestrantes; } \\
\text { - Os alunos também falaram sobre aprender com histórias de pacientes. }\end{array}$ \\
\hline $\begin{array}{l}\text { El Hussein, Salyers, \& } \\
\text { Osuji, } 2015 .\end{array}$ & ND & ND & Foi utilizado ilustração narrativa visual. & $\begin{array}{l}\text { O assunto foi melhor absorvido; } \\
\text { Os alunos conseguem lembrar das ilustrações ao discutir conceitos com o corpo } \\
\text { docente clínico em situações de estresse. }\end{array}$ \\
\hline Fenton, 2014. & 40 & $\begin{array}{l}\text { Especialização } \\
\text { enfermagem infantil e } \\
\text { juvenil; último ano. }\end{array}$ & $\begin{array}{l}\text { Os alunos podem navegar pelo objeto de } \\
\text { aprendizagem visualizando imagens da } \\
\text { jovem e ouvindo sua narrativa, dentro do } \\
\text { ambiente virtual Blackboard. }\end{array}$ & $\begin{array}{l}\text { - O recurso permitiu obter a perspectiva de um jovem e } 80 \% \text { dos alunos o classificou } \\
\text { - } \quad \text { A descrição da inexperiência das enfermeine do conteúdo; } \\
\text { interessante para os estudantes que pretendem atuar na área. }\end{array}$ \\
\hline Paliadelis et al., 2014. & 284 & ND & $\begin{array}{l}\text { Realizadas através de dramas de áudio, } \\
\text { inseridos em um programa de } \\
\text { aprendizagem online. }\end{array}$ & $\begin{array}{l}\text { - As atividades foram agradáveis e permitiram adquirir conhecimentos e melhorias } \\
\text { acerca da prática; } \\
\text { O poder da narrativa envolveu, promoveu a aprendizagem reflexiva e possibilitou a } \\
\text { imaginação de uma forma diferente de trabalhar. }\end{array}$ \\
\hline Gidman, 2013. & 12 & $\begin{array}{l}\text { Pós-graduação } \\
\text { enfermagem (6), } \\
\text { especialização obstetrícia } \\
\text { (3), pós-graduação } \\
\text { trabalho social (3); último } \\
\text { ano. }\end{array}$ & $\begin{array}{l}\text { As narrações foram realizadas pelos } \\
\text { pacientes durante os estágios de prática. }\end{array}$ & $\begin{array}{l}\text { - Ocorreu estimulo da aprendizagem em relação a uma variedade de experiências e } \\
\text { demonstrou valor como conhecimento para prática; } \\
\text { Desenvolveu-se relacionamento e apoio mútuo com os pacientes, permitindo } \\
\text { aprender sobre perspectivas subjetivas e emocionais; } \\
\text { A aprendizagem não foi ativamente facilitada por educadores, e no geral, não houve } \\
\text { chance de refletir com profissionais experientes. }\end{array}$ \\
\hline Bradshaw, 2012. & 24 & $\begin{array}{l}\text { Curso de preceptoria em } \\
\text { enfermagem; sexto } \\
\text { semestre. }\end{array}$ & $\begin{array}{l}\text { As histórias foram lidas num clube do livro } \\
\text { e escritas pelos alunos. }\end{array}$ & $\begin{array}{l}\text { - Através do feedback dos alunos, houve relatos que a história narrada acaba trazendo } \\
\text { preferência do aluno por uma aprendizagem profunda. }\end{array}$ \\
\hline Stacey \& Hardy, 2011. & 58 & $\begin{array}{l}\text { Graduação; último } \\
\text { módulo. }\end{array}$ & $\begin{array}{l}\text { As histórias digitais ocorreram através de } \\
\text { curta-metragem num fórum educacional } \\
\text { com workshops. }\end{array}$ & $\begin{array}{l}\text { - O conteúdo emocional apresenta um desafio para os facilitadores; } \\
\text { A autenticidade das histórias permitiu se relacionar com o contador que modelava o } \\
\text { processo reflexivo, permitindo observar sua ação e se colocar como recém-formado; } \\
\text { - Os alunos reconheceram o levantamento de questões preocupantes. }\end{array}$ \\
\hline Christiansen, 2011. & 20 & $\begin{array}{l}\text { Graduação; terceiro } \\
\text { período. }\end{array}$ & $\begin{array}{l}\text { Realizada através de curtas apresentações } \\
\text { multimídias com imagens e músicas. }\end{array}$ & $\begin{array}{l}\text { - A história multimídia é mais favorável que o texto escrito, porém menos poderosa } \\
\text { - } \quad \text { Houve ouvir o paciente em sala de aula; } \\
\text { relevância; } \\
\text { - Foi estabelecida relação empática entre o contador e o ouvinte; } \\
\text { - Houve desconforto ao confrontar a perspectiva do contador. Para alguns alunos, } \\
\text { serviu como gatilho para a reflexão crítica; } \\
\text { - Ocorreu engajamento crítico e novos insights sobre si e sua prática. }\end{array}$ \\
\hline Nicholson, 2010. & 74 & $\begin{array}{l}\text { Graduação; segundo } \\
\text { semestre. }\end{array}$ & $\begin{array}{l}\text { Realizada de forma verbal pelo professor, } \\
\text { durante a simulação. }\end{array}$ & $\begin{array}{l}\text { A pedagogia narrativa permitiu fornecer feedback imediato do desempenho e } \\
\text { tomada de decisão; } \\
\text { A simulação com pedagogia narrativa permite ao professor orientar a discussão e } \\
\text { reflexão de decisões, e forneceu mais interação aluno-professor e colaboração entre } \\
\text { colegas. }\end{array}$ \\
\hline $\begin{array}{l}\text { Baguley \& Brown, } \\
2009 .\end{array}$ & 11 & $\begin{array}{l}\text { Pós-graduação educação } \\
\text { (4), pós-graduação }\end{array}$ & $\begin{array}{l}\text { A narração foi feita de forma escrita entre } \\
\text { os estudantes de cada curso, com feedback. }\end{array}$ & $\begin{array}{l}\text { - A troca de narrativas demonstrou sentimentos de empatia, acordo, conexão, } \\
\text { qualificação/desafios e desenvolveu pensamentos crítico-reflexivos; } \\
\text { - O feedback empático foi maior nos alunos da pós-graduação de enfermagem; }\end{array}$ \\
\hline
\end{tabular}


Research, Society and Development, v. 10, n. 15, e580101523185, 2021

(CC BY 4.0) | ISSN 2525-3409 | DOI: http://dx.doi.org/10.33448/rsd-v10i15.23185

\begin{tabular}{|c|c|c|c|c|}
\hline & & $\begin{array}{l}\text { enfermagem (7); primeiro } \\
\text { ano. }\end{array}$ & & - $\quad$ Adquiriu-se novos conhecimentos sobre a experiência no outro curso. \\
\hline $\begin{array}{l}\text { Ekebergha, Leppb, \& } \\
\text { Dahlberga, } 2004 .\end{array}$ & ND & ND & Realizada através de dramatização. & $\begin{array}{l}\text { Ao trabalhar com experiências usando drama, reflexão e expressões verbais, } \\
\text { esclareceram-se sentimentos e imaginações; } \\
\text { Desenvolveu-se uma visão sobre como a teoria do cuidado pode se tornar uma } \\
\text { ferramenta no contexto da prática. }\end{array}$ \\
\hline Ironside, 2003. & 14 & $\begin{array}{l}\text { Graduação; primeiro } \\
\text { semestre. }\end{array}$ & Realizadas de forma oral e escritas. & $\begin{array}{l}\text { - Através do curso com abordagem de pedagogia narrativa, os alunos passaram a } \\
\text { entender a enfermagem de forma diferente; } \\
\text { - A pedagogia narrativa proporcionou um ambiente propicio à aprendizagem e } \\
\text { permitiu a livre participação. }\end{array}$ \\
\hline Platt, 2002. & ND & ND & Realizadas de forma verbal em grupo. & $\begin{array}{l}\text { - As histórias às vezes têm a capacidade de alto impacto emocional e podem ser } \\
\text { desconcertantes. }\end{array}$ \\
\hline Cooper, 2000. & ND & ND & $\begin{array}{l}\text { Através da apresentação de um cenário, as } \\
\text { narrativas foram feitas de forma escrita e } \\
\text { compartilhadas entre os grupos. }\end{array}$ & $\begin{array}{l}\text { - } \quad \text { Desenvolveu-se habilidades de raciocínio e compreensão mais profunda; } \\
\text { - } \quad \text { Puposições desafiadoras e abertura a outras ideias; } \\
\text { confiança. }\end{array}$ \\
\hline Wilkinson et al., 1998. & 97 & Graduação; primeiro ano. & $\begin{array}{l}\text { Realizada de forma verbal na sala de aula e } \\
\text { em ambientes clínicos por pacientes, } \\
\text { enfermeiras, palestrantes, colegas } \\
\text { estudantes e professor. }\end{array}$ & $\begin{array}{l}\text { - As histórias das enfermeiras na prática apresentaram o mundo da enfermagem e } \\
\text { facilitaram o sentimento de pertencimento; } \\
\text { - Ocorreu integração entre teoria e prática; } \\
\text { - Palestrantes mais 'divertidos' tiveram mais sucesso em garantir e manter a atenção } \\
\text { do aluno; } \\
\text { - Despertou-se emoção no apresentador e ouvinte, permitindo melhor conexão com o } \\
\text { paciente; } \\
\text { Foi fornecido contexto e permitido o envolvimento ativo dos alunos; } \\
\text { O que foi aprendido foi usado para crescimento pessoal e para a preparação para a } \\
\text { enfermagem. }\end{array}$ \\
\hline
\end{tabular}

ND - Não descrito. Fonte: Autores (2021). 


\section{Discussão}

O mapeamento das evidências relacionadas a utilização da narração de histórias como estratégia de aprendizagem na formação do enfermeiro permitiu a identificação dos países de origem das produções científicas, benefícios e desvantagens da metodologia, temas abordados e desempenho dos recursos utilizados. A variabilidade dos métodos de pesquisa dos estudos apresentou uma diversificada gama de achados referentes a esses aspectos.

No que diz respeito às origens das publicações, foi possível observar posição de destaque para o Reino Unido, que contribuiu com 38\% das pesquisas referentes à temática, seguido pelos Estados Unidos com um percentual de 28,6\%. Esses achados se correlacionam com estudo recente que identifica essas nações entre as cinco maiores produtoras de artigos na área da enfermagem no mundo, ocupando o terceiro e primeiro lugar, respectivamente. Outros países identificados na presente revisão se encontram entre essas posições, sendo a Austrália ocupante da segunda e o Canadá da quinta colocação (Silva \& Vanz, 2021).

Ao que se refere aos benefícios, nota-se uma confluência de algumas das descobertas encontradas nesta revisão com uma recente pesquisa, podendo destacar: envolvimento e captação da atenção, formação de elo entre teoria e prática clínica, melhora da compreensão, aumento da empatia, favorecimento do estabelecimento de uma identidade de enfermagem, demonstração da atualidade clínica, capacitação e promoção da profissão (Attenborough \& Abbott, 2020).

Outros benefícios oriundos da presente análise podem-se somar aos anteriormente mencionados, sendo eles, desenvolvimento de raciocínio, reflexão crítica e imaginação; aumento da confiança na atuação clínica real; promoção de contexto, engajamento e novos insights; conexão interpessoal; melhora da competência clínica; aprendizagem profunda, cooperativa e subjetiva; exploração de temas pertinentes; aquisição de conhecimentos e percepções; retenção de conteúdo; fornecimento de feedback de desempenho e tomada de decisão; e possibilidade de orientação de discussões e crescimento pessoal.

A partir disso e da análise da literatura, percebe-se que a narração de histórias possibilita uma variedade de vantagens para a formação diferencial do profissional de enfermagem, proporcionando assim uma visão integral, diferenciada e humanizada do paciente (Silva \& Sei, 2019). O desenvolvimento do sentimento empático advindo dessa metodologia é o principal responsável por fomentar essas contribuições, principalmente a humanização em saúde (Silva \& Sei, 2019), sendo esta requerida pelas DCNs do curso de enfermagem (Brasil, 2001).

Apesar das inúmeras vantagens relatadas, é válido ressaltar que mesmo pontuais, foram encontradas algumas desvantagens na aplicação da metodologia. As mesmas estão atreladas ao impacto emocional das histórias e se associam em sua maioria ao papel do contador de histórias. À medida que promove empatia aos alunos, pode provocar no contador de histórias vulnerabilidade (Urstad et al., 2018), desconforto (Christiansen, 2011) e se apresentar como um desafio (Stacey \& Hardy, 2011). Para ambos, educando e contador, o impacto emocional da história pode ser desconcertante (Platt, 2002).

Ainda, evidenciou-se que o uso da narração de histórias ocorre em diferentes contextos de aprendizagem, ao trabalhar temas relacionados a experiências clínicas, histórias subjetivas, diferentes áreas do conhecimento e conteúdo específico, como cardiologia, conceito de fisiopatologia, obstetrícia, saúde mental e cuidados paliativos e/ou no fim da vida. Outros estudos corroboram e contribuem para essa variedade de temáticas, a exemplo das pesquisas de Bachur et al. (2019) e Félix e Soares (2019) que se referem a contação de histórias no ensino de disciplinas específicas.

No contexto educacional, essas variedades temáticas justificam-se pelos objetivos do uso da narração de histórias apresentarem-se variados. Os mesmos podem possuir como finalidades a promoção da reflexão do aluno, discussão, práticas de ensino, compartilhamento de experiências e auxilio em outras metodologias ativas, como por exemplo associado à simulação (Mojtahedzadeh, Mohammadi, Emami, \& Zarei, 2021; Palácio, Ciannella, \& Struchiner, 2017).

É importante notar que diferentes tipos de recursos foram utilizados para aplicar a narração, e isso contribuiu para a variedade de achados discutidos até então. Em sua maioria foram utilizadas mídias digitais, muitas delas com caráter multimídia, 
caracterizando-as como narrativas digitais (Lambert \& Hessler, 2018). Os resultados encontrados nos estudos desta revisão demonstram que os desempenhos dos recursos apresentaram diferentes impactos na aprendizagem dos educandos, com um menor desempenho atrelado ao podcast (Conlon et al., 2020) e ao texto escrito (Christiansen, 2011; Cooper, 2000).

Os melhores resultados se associaram ao uso de meios multimídias com áudio e imagem, promovendo o desenvolvimento de uma aprendizagem efetiva e consolidada, como visto nos estudos de Conlon et al. (2020), Beck e Sitzman (2019), Urstad et al. (2018), Johnston et al. (2017), Scamell e Hanley (2017), Price et al. (2015), Fenton (2014), Stacey e Hardy (2011), Christiansen (2011). Dessa forma, percebe-se que as narrativas digitais podem ser utilizadas para transformar o processo didático na enfermagem, com vistas a uma formação eficaz.

Também foi possível notar que as narrações realizadas através da forma verbal apresentaram resultados significativos (Hold et al., 2015; Gidman, 2013; Bradshaw, 2012; Ironside, 2003; Platt, 2002; Wilkinson et al., 1998). Isso indica que apesar do método da oralidade ser o mais ancestral para se narrar histórias, ainda se mostra eficiente e possui espaço de aplicação no ensino superior.

Apesar dos diferentes desempenhos, todos os recursos proporcionaram achados acerca do uso da narração de histórias na educação em enfermagem. O conhecimento desse variado leque de opções para a implementação da metodologia serve como subsídio para os educadores. Os mesmos necessitam estar capacitados para seu uso, no intuito de incorporar uma estratégia ativa de ensino-aprendizagem, visto que esse é um dos grandes desafios da aprendizagem no século XXI (Félix \& Soares, 2019).

Assim, torna-se evidente que a narração de histórias tem sido implementada no ensino da enfermagem nas últimas duas décadas e apresenta múltiplas vantagens para a formação do enfermeiro. É válido ressaltar que para a implementação da narração ser bem-sucedida, o educador contador de histórias deve conhecer seu público alvo, considerando os grupos sociais, interesses, faixas etárias e seus feedbacks com relação a aceitação da metodologia empregada (Valença \& Tostes, 2019).

\section{Considerações Finais}

A presente revisão de escopo identificou o conhecimento produzido acerca da utilização da narração de histórias como estratégia na formação do enfermeiro. Para sua efetividade, o conteúdo da narrativa e os recursos utilizados mostram-se como atributos importantes, e os benefícios do seu uso vão desde desenvolvimento e/ou aumento da empatia à captação da atenção, formação do elo teoria-prática, melhora da compreensão e capacitação.

Como limitações encontradas neste estudo, apontam-se a indisponibilidade na íntegra de alguns artigos elencados durante a seleção e as diminutas amostras utilizadas nas pesquisas conduzidas sobre a temática em questão. Neste sentido, sugerese que pesquisas futuras ampliem as amostras estudadas na avaliação dessa intervenção no ensino de enfermagem, de maneira que os dados componham uma base sólida que promova a validação dessa ferramenta.

\section{Referências}

Attenborough, J., \& Abbott, S. (2020). Using storytelling in nurse education: The experiences and views of lecturers in a higher education institution in the United Kingdom. Nurse Education in Practice.

Bachur, T. P. R., Freire, R. M., Sibellino, L. O., Pantoja, L. D. M., Braga, C. J. M., \& Aragão, G. F. (2019). Paródias e contação de história: formas lúdicas de ensinar parasitologia no ensino superior. Revista de Ciências Médicas e Biológicas, 18(1), 79-88.

Baguley, M., \& Brown, A. (2009). Critical friends: an investigation of shared narrative practice between education and nursing graduates. Teaching in Higher Education, 14(2), 195-207.

Beck, M. S., \& Sitzman, K. (2019). Compelling reasons for using digital stories to teach: a descriptive qualitative study. Teaching and Learning in Nursing, 14(4), 265-269.

Bradshaw, V. (2012). The Effect of Teaching with Stories on Associate Degree Nursing Students' Approach to Learning and Reflective Practice (Dissertation Doctorate degree). Education, Arizona State University. 
Brasil. (2001). Ministério da Educação. Conselho Nacional de Educação. Câmara de Educação Superior. Resolução CNE/CES N. 3, de 07 de novembro de 2001. Institui as Diretrizes Curriculares Nacionais do Curso de Graduação em Enfermagem. Diário Oficial da República Federativa da União. Brasília, 09 nov. 2001. Seção 1, p. 37. Brasília (DF): Ministério da Educação e Cultura. http://portal.mec.gov.br/cne/arquivos/pdf/CES03.pdf

Brasil. (2018). Ministério da Saúde. Conselho Nacional de Saúde. Resolução n ${ }^{o}$ 573, de 31 de janeiro de 2018. Aprova o Parecer Técnico nº 28/2018 contendo recomendações do Conselho Nacional de Saúde (CNS) à proposta de Diretrizes Curriculares Nacionais (DCN) para o curso de graduação Bacharelado em Enfermagem. Brasília-DF. https://www.in.gov.br/materia/-/asset_publisher/Kujrw0TZC2Mb/content/id/48743098/do1-2018-11-06-resolucao-n-573-de-31-dejaneiro-de-2018-48742847

Camargo, F., \& Daros, T. (2018). A sala de aula inovadora-estratégias pedagógicas para fomentar o aprendizado ativo. Porto Alegre: Penso Editora.

Christiansen, A. (2011). Storytelling and professional learning: A phenomenographic study of students' experience of patient digital stories in nurse education. Nurse education today, 31(3), 289-293.

Cooper, N. J. (2000). The use of narrative in the development of critical thinking. Nurse Education Today, 20(7), 513-518.

Collins, S. N. (2021). The importance of storytelling in chemical education. Nature Chemistry, 13(1), 1-2.

Conlon, M. M., Smart, F., \& Mcintosh, G. (2020). Does technology flatten authenticity? Exploring the use of digital storytelling as a learning tool in mental health nurse education. Technology, Pedagogy and Education, 29(3), 269-278.

Edwards, S. L. (2016). Narrative analysis: How students learn from stories of practice. Nurse Researcher, 23(3), 18-25.

Ekebergh, M., Lepp, M., \& Dahlberg, K. (2004). Reflective learning with drama in nursing education-a Swedish attempt to overcome the theory praxis gap. Nurse Education Today, 24(8), 622-628.

El hussein, M. T., Salyers, V., \& Osuji, J. (2016). Use of visual narrative illustrations to teach pathophysiology concepts to nursing students. Journal of Nursing Education, 55(2), 109-112.

Felix, A. M. S., \& Soares, R. A. Q. (2019). Metodologias ativas no ensino de enfermagem em doenças transmissíveis. Rev. enferm. UFPE on line, 13, e241816.

Fenton, G. (2014). Involving a young person in the development of a digital resource in nurse education. Nurse education in practice, 14(1), 49-54.

Gidman, J. (2013). Listening to stories: Valuing knowledge from patient experience. Nurse education in practice, 13(3), 192-196.

Hold, J. L., Blake, B. J., \& Ward, E. N. (2015). Perceptions and experiences of nursing students enrolled in a palliative and end-of-life nursing elective: A qualitative study. Nurse education today, 35(6), 777-781.

Ironside, P. M. (2003). Trying something new: Implementing and evaluating narrative pedagogy using a multimethod approach. Nursing education perspectives, 24(3), 122-128.

Johnston, S.; Parker, C. N.; \& Fox, A. (2017). Impact of audio-visual storytelling in simulation learning experiences of undergraduate nursing students. Nurse education today, 56, 52-56.

Lambert, J., \& Hessler, B. (2018). Digital Storytelling Capturing Lives, Creating Community (5th ed). Routledge. 234 p.

Mclellan, H. (2007). Digital storytelling in higher education. Journal of Computing in Higher Education, 19(1), 65-79.

Mojtahedzadeh, R., Mohammadi, A., Emami, A. H., \& Zarei, A. (2021). How Digital Storytelling Applied in Health Profession Education: A Systematized Review. Journal of Advances in Medical Education \& Professionalism, 9(2), 63.

Nicholson, A. C. (2010). Comparison of selected outcomes based on teaching strategies that promote active learning in nursing education (Thesis Doctorate degree). Philosophy degree in Educational Policy and Leadership Studies, Graduate College of The University of Iowa, Estados Unidos.

Palácio, M. A. V., Ciannella, D., \& Struchiner, M. (2017). Narrativas Digitais e Aprendizagem: um panorama a partir do ensino da saúde. Revista Eletrônica de Comunicação, Informação e Inovação em Saúde, 11(2), 1-15.

Paliadelis, P. S., Stupans, I., Parker, V., Piper, D., Gillan, P., Lea, J., ... \& Fagan, A. (2015). The development and evaluation of online stories to enhance clinical learning experiences across health professions in rural Australia. Collegian, 22(4), 397-403.

Peters, M. D., Godfrey, C. M., Khalil, H., McInerney, P., Parker, D., \& Soares, C. B. (2015). Guidance for conducting systematic scoping reviews. JBI Evidence Implementation, 13(3), 141-146.

Platt, C. (2002). Nurses' Fit for Purpose': Using a task-centred group to help students learn from experience. Teaching in Higher Education, 7(1), 33-45.

Price, D. M., Strodtman, L., Brough, E., Lonn, S., \& Luo, A. (2015). Digital storytelling: an innovative technological approach to nursing education. Nurse educator, 40(2), 66-70.

Roman, C., Ellwanger, J., Becker, G. C., Silveira, A. D. D., Machado, C. L. B., \& Manfroi, W. C. (2017). Metodologias ativas de ensino-aprendizagem no processo de ensino em saúde no Brasil: uma revisão narrativa. Clinical and biomedical research, 37(4), 349-357.

Santos, R. C. A. L. (2020). Reflexões sobre a arte de contar histórias. Revista Educação Pública, 20(5).

Saraiva, K., Traversini, C. S., \& Lockmann, K. (2020). A educação em tempos de COVID-19: ensino remoto e exaustão docente. Práxis educativa, 15(e2016289), 1-24. 
Research, Society and Development, v. 10, n. 15, e580101523185, 2021

(CC BY 4.0) | ISSN 2525-3409 | DOI: http://dx.doi.org/10.33448/rsd-v10i15.23185

Scamell, M., \& Hanley, T. (2017). Innovation in preregistration midwifery education: Web based interactive storytelling learning. Midwifery, 50, 93-98.

Silva, A. C. M., \& Sei, M. B. (2019). A Contação de Histórias e a humanização no hospital: vivências de profissionais da saúde. Revista da SBPH, 22(2), 6889

Silva, R. C., Fº, \& Vanz, S. A. S. (2021). Análise da produção científica da Enfermagem e seus leitores no Mendeley. Revista Ibero-Americana de Ciência da Informação, 14(1), 215-237.

Stacey, G., \& Hardy, P. (2011). Challenging the shock of reality through digital storytelling. Nurse education in practice, 11 (2), $159-164$.

Treloar, A., Mcmillan, M., \& Stone, T. (2017). Nursing in an imperfect world: Storytelling as preparation for mental health nursing practice: Storytelling as preparation for practice. International Journal of Mental Health Nursing, 26(3), 293-300.

Tricco, A. C., Lillie, E., Zarin, W., O'Brien, K. K., Colquhoun, H., Levac, D., ... \& Straus, S. E. (2018). PRISMA extension for scoping reviews (PRISMA$\mathrm{ScR})$ : checklist and explanation. Annals of internal medicine, 169(7), 467-473.

Urstad, K. H., Ulfsby, K. J., Brandeggen, T. K., Bodsberg, K. G., Jensen, T. L., \& Tjoflåt, I. (2018). Digital storytelling in clinical replacement studies: Nursing students' experiences. Nurse education today, 71, 91-96.

Valença, M. M., \& Tostes, A. P. B. (2019). O Storytelling como ferramenta de aprendizado ativo. Rev. Carta Internacional, 14(2), 221-243.

Wilkinson, C., Peters, L., Mitchell, K., Irwin, T., McCorrie, K., \& MacLeod, M. (1998). 'Being there': learning throughactive participation. Nurse education today, 18(3), 226-230. 